\title{
Adaptive mathematical model of tumor response to radiotherapy based on CBCT data
}

\author{
A. Belfatto, M. Riboldi, D. Ciardo, A. Cecconi, R. Lazzari, B.A. Jereczek-Fossa, \\ R. Orecchia, G. Baroni, P. Cerveri
}

\begin{abstract}
Mathematical modeling of tumor response to radiotherapy has the potential of enhancing the quality of the treatment plan, which can be even tailored on an individual basis. Lack of extensive in vivo validation has prevented, however, reliable clinical translation of modeling outcomes. Image guided radiotherapy (IGRT) is a consolidated treatment modality based on computed tomographic (CT) imaging for tumor delineation and volumetric cone beam CT data for periodic checks during treatment. In this work, a macroscopic model of tumor growth and radiation response is proposed, being able to adapt along the treatment course as volumetric tumor data become available. Model parameter learning was based on cone beam $\mathrm{CT}$ images in 13 uterine cervical cancer patients, subdivided into three groups $($ G1, G2, G3) according to tumor type and treatment. Three groupspecific parameter sets (PS1, PS2 and PS3) on one general parameter set (PSa) were applied. The corresponding average model fitting errors were $14,18,13$ and $21 \%$, respectively. The model adaptation testing was performed using volume data of three patients, other than the ones involved in the parameter learning. The extrapolation performance of the general model was improved, while comparable prediction errors were found for the groupspecific approach. This suggests that an on-line parameter tuning can overcome the limitations of a suboptimal patient stratification, which appeared otherwise a critical issue.
\end{abstract}

Index Terms - Mathematical model, tumor growth, parameter adaptation, radiation therapy, IGRT

\section{INTRODUCTION}

$\mathrm{U}$ TERINE cervical cancer is one of the deadliest disease for women worldwide, with about 270,000 deaths annually [1]. The mortality rate in the United States is over $30 \%$ within five years from diagnosis [2]. External beam radiotherapy (EBRT), often administered together with adjuvant chemotherapy, represents a highly effective localized treatment for such a tumor, in which image guided radiotherapy (IGRT) plays a key role [3] [4]. IGRT

Research supported by Italian Ministry of Education, University and Research.

A. Belfatto, M. Riboldi, G. Baroni, P. Cerveri are with the Department of Electronics, Information and Bioengineering - Politecnico di Milano University - Milan, Italy (corresponding author: A. Belfatto; e-mail: belfatto.antonella@googlemail.com) encompasses the acquisition of morphological images at each treatment fraction, allowing accurate patient set up and, potentially, treatment re-planning [5] [6]. In this perspective, IGRT, along with prognostic mathematical tools, discloses the opportunity of daily treatment adaptation according to measured tumor evolution (plan of the day) [4] [6] [7].

Cell abnormal growth, due to genetic mutations and deregulation of control mechanisms, and the response to radiation therapy, involve complex events at different spatial and temporal scales [8] [9] [10]. Macroscopic indices, as the FIGO (International Federation of Gynecology and Obstetrics) staging, which is mainly determined by the tumor extent, lymph node status and metastasis occurrence, have been proved to be strongly correlated with the patient prognosis [2]. Cell doubling time as a sign of tumor aggressiveness [8], volume decrease as an indicator of treatment effectiveness [11], and tumor oxygenation as a sign of tumor radioresponsiveness [12], have been more recently drawing the attention of oncologists and radiotherapists. A more extensive approach, to elucidate the tumor evolution and interaction with the treatment, is represented by mathematical models. They enable the in-silico simulation of various biological processes underlying both cell abnormal growth, and the response to radio- and chemo-therapy. Complex mechanistic models, featuring both spatial and temporal dynamics at molecular and cellular scales, have been extensively investigated [13] [14] [15]. Despite their fine approximation to reality, such models have been hardly applied in clinical practice, as the detailed morphologic and functional patient data required for their use and validation is prevented mainly by the invasiveness and cost of the experimental procedures entailed.

Alternatively, simpler models at the tissue scale, representing tumor growth under conditions of mechanical pressure, nutrient shortage, and therapeutic irradiation, have been proposed. It has been widely reported that macroscopic model parameters can be directly linked to actual measurements obtained from clinical images, such as computed tomography (CT) and magnetic resonance imaging (MRI) [16] [17] [18] [19] [20] [21] [22] [23].

Some works have focused on numerical simulations [19], whereas others have proposed patient-specific data fitting and correlation analysis between model parameters and therapy

A. Ceccone, D. Ciardo, R. Lazzari, B.A. Jereczek, R. Orecchia are with Advanced Radiotherapy Center - European Institute of Oncology - Milan, Italy. 
outcome [16] [18]. In a few cases, the model was trained on a group-specific basis to predict the tumor response during treatment [17] [21]. In [20], the parameters were set according to previous literature findings and the model was validated on a small dataset of patients. Our previous models [21] [22] [23] showed promising results with an average fitting error value as low as $5 \%$, while analysis of their extrapolation abilities was not conclusive. On the one hand, it was acknowledged that parameter setting, according to literature values as well as in vitro studies, could lead to large prediction errors. On the other hand patient-specific approaches may lack of generalization ability [21] [22] [23] [24] [25] [26]. A challenging opportunity is represented by the adaptation of the model parameters at run-time during treatment using patient-specific measurements, which, in principle, could improve the model prediction reliability and trigger possible therapy adjustments in terms of fractionation and dose [5] [6].

This study proposes a novel discrete-time mathematical model, accounting for the evolution of active, inactive and necrotic tumor volumes. It combines the accuracy of the patient-specific approach and the potential extrapolation ability of the group-specific optimization by means of an adaptation strategy able to refine model parameters along the treatment course. The validation of predictive ability is presented, as applied on uterine cervical cancer patients administered with radiation therapy.

\section{MATERIALS AND METHODS}

\section{A. Patient data}

Sixteen women affected by uterine cervix cancer were retrospectively included in three subgroups $(\mathrm{G} 1, \mathrm{G} 2$ and G3) according to the tumor type and administered therapy; none of them underwent surgery as treatment option. Six older patients (mean age: 83) affected by squamous cell carcinoma (SCC) and belonging to the first group $(\mathrm{G} 1)$, were treated by means of external beam radiation therapy (EBRT) using the Trilogy ${ }^{\circledR}$ system (VARIAN medical systems, Palo Alto, California, USA). The second group (G2) consisted of five patients (mean age: 46). affected by adenocarcinoma (ADC). Their treatment included concurrent EBRT and adjuvant chemotherapy. Finally, data from five women (mean age: 52) affected by squamous cell carcinoma and administered with chemotherapy during EBRT, were gathered in G3 (Table I). G2 and G3 patients received Cisplatin and Paclitaxel once a week (46 cycles). After the external radiation treatment, all the patients underwent internal brachytherapy (4-5 sessions). All the patients were treated between 2010 and 2012 at the European Institute of Oncology (IEO, Milan - Italy). Among them, two died due to cancer-unrelated causes, the others survived up to 24-months follow-up. Patients received up to 50.4 Gy in 23 to 28 fractions with a 5-day per week schedule (Table I). The treatment started within two weeks from the planning CT acquisition, which was used to simulate the dose distribution (Fig.1). Patient set-up was verified and corrections were performed according to IEO institutional protocols, at each radiotherapy session. The images were acquired by means of a $\mathrm{kV}$ CBCT system featuring a voxel dimension of $0.87 \times 0.87 \times 3 \mathrm{~mm}^{3}$. The gross tumor volume (GTV) was manually contoured on a subset of the acquired CBCT and CT scans by an expert radiation oncologist (Fig.1), using the commercial software MIMvista (MIMvista Corporation, Cleveland, OH, USA). The same software reconstructed automatically the tumor volume from the contours. The number of slices varied intra- and inter- patient as a function of the tumor size. The average volume throughout the patient cohort was on average $23.7 \mathrm{~cm}^{3}$ (range: $12.7-44.4 \mathrm{~cm}^{3}$ ) at the beginning of radiotherapy (first CBCT) and $8.6 \mathrm{~cm}^{3}$ (range: 3.6-17.1 $\mathrm{cm}^{3}$ ) at the end of radiotherapy (last CBCT), respectively.

TABLE I

PATIENT DATA SUMMARY. RT INDICATES THE ADMINISTRATION OF RADIATION THERAPY ONLY, WHILE RT+CHT CORRESPONDS TO THE COMBINATION OF RADIOTHERAPY AND CHEMOTHERAPY. THE DOSE IS EXPRESSED IN GY/FRACTION AND \#CBCT REPRESENTS THE AMOUNT OF SEGMENTED CBCTS.

\begin{tabular}{|lllllll|}
\hline \hline Code & Group & Tumor & Therapy & Fractions & Dose & \#CBCT \\
\hline P1 & G1 & SCC & RT & 25 & 2 & 8 \\
P2 & G1 & SCC & RT & 25 & 2 & 6 \\
P3 & G1 & SCC & RT & 25 & 1.8 & 7 \\
P4 & G1 & SCC & RT & 28 & 1.8 & 8 \\
\hline P5 & G1 & SCC & RT & 28 & 1.8 & 27 \\
\hline P6 & G1 & SCC & RT & 25 & 1.8 & 6 \\
P7 & G2 & ADC & RT+ChT & 25 & 2 & 9 \\
P8 & G2 & ADC & RT+ChT & 25 & 2 & 11 \\
\hline P9 & G2 & ADC & RT+ChT & 25 & 2 & 10 \\
\hline P10 & G2 & ADC & RT+ChT & 25 & 2 & 9 \\
P11 & G2 & ADC & RT+ChT & 28 & 1.8 & 12 \\
P12 & G3 & SCC & RT+ChT & 28 & 1.8 & 7 \\
P13 & G3 & SCC & RT+ChT & 23 & 2 & 7 \\
P14 & G3 & SCC & RT+ChT & 25 & 2 & 6 \\
P15 & G3 & SCC & RT+ChT & 25 & 2 & 8 \\
\hline P16 & G3 & SCC & RT+ChT & 28 & 1.8 & 28 \\
\hline \hline
\end{tabular}

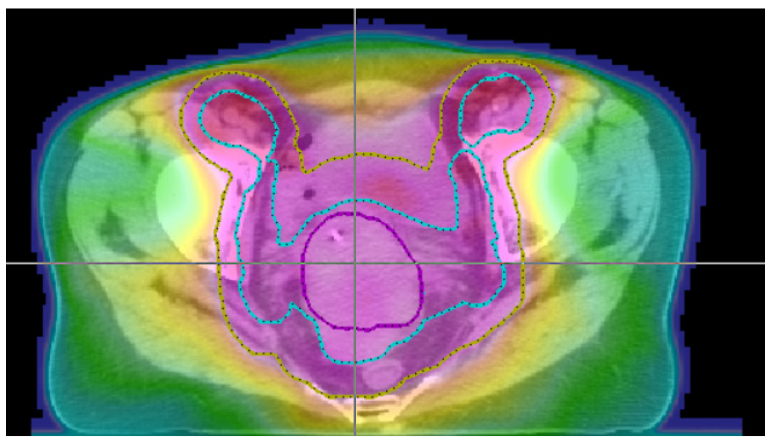

Fig. 1. Example of pre-treatment CT including the delineation of the gross tumor volume (GTV) in violet and of the clinical target volume (CTV) in cyan. The dose profile is defined by means of iso-dose regions. The pink one, were the total amount of dose is delivered, is almost superimposed to the planning target volume (PTV, yellow line)

\section{B. Mathematical model}

Three different tumor dynamics were modeled throughout discrete time (1-day resolution): 1) active volume $\left(V_{a}\right)$, 
featuring the growth of the undamaged tumor; 2) inactive volume $\left(V_{i}\right)$, that is the fraction of the active volume impaired by treatment; 3$)$ necrotic volume $\left(V_{n}\right)$, that is the inactive volume portion permanently necrotized and undergoing reabsorption. The $V_{a}$ dynamics was driven by the surviving fraction $S F$, which represents the percentage of the tumor volume that is not irreversibly damaged by the irradiation dose $d$. The $S F$ has been traditionally represented by the linear quadratic (LQ) exponential model [16] [20] [22] [23] as:

$S F=e^{-\left(\alpha d+\beta d^{2}\right)}$

where $\alpha\left(\mathrm{Gy}^{-1}\right)$ and $\beta\left(\mathrm{Gy}^{-2}\right)$ are cell- and radiation-specific parameters referring to lethal (double-strand damage) and sublethal (reparable or single strand damage), respectively [26]. In the present development, we hypothesized an explicit relationship between $\alpha$ and the oxygenation level $p O_{2}$ so that eq. (1) can be rearranged as:

$S F=e^{-\alpha\left(p O_{2}\right)\left(d(t)+\frac{d(t)^{2}}{10}\right)}$

where $d(t)$ is equal to the irradiated dose value at the time of irradiation and null elsewhere. The $\alpha / \beta$ ratio was set equal to 10 as suggested in the literature [16]. The repopulation was represented by the Gompertian function through the growth rate $(\rho)$ and the carrying capacity $(k)$, this last corresponding to the maximum active volume size [21]. Incorporating the $S F$ and the repopulation, $V_{a}$ dynamics can be finally written as:

$V_{a}(t+1)=V_{a}(t)\left(1+\rho \log \left(\frac{k}{V_{a}(t) \mathrm{SF}}\right)\right) S F$

with $S F=1$ for null dose. The radiation therapy damage (e.g. strand breaks) can result both in cell death or in cell inability to duplicate [27]. The inactivated volume, $V_{i}$, represents the cells irreversibly impaired, which do not contribute any longer to the tumor growth but at the same time do not undergo the clearance process until their cell-cycle (about 1 day) is completed. According to these observations, we assumed that the severely damaged cells turn into necrotic bulk within two days from irradiation. Hence, the inactivated volume dynamics can be written as:

$V_{i}(t+1)=(1-r) V_{a}(t)(1-S F)$

where the parameter $r$ represents the percentage of cells that are going to die within the first day, whereas $1-r$ is the fraction of cells that survived from 1 up to 2 days after irradiation.

The necrotic volume portion $V_{n}$, embedding the instantaneous cell apoptosis and the delayed death of the tumor portion inactivated by the dose of the previous day, was modeled through an exponential decay as

$$
\begin{aligned}
V_{n}(t+1) & =V_{n}(t) e^{-\frac{\log (2)}{T_{1 / 2}}\left(t-t_{r}\right)}+V_{i}(t) e^{-\frac{\log (2)}{T_{1 / 2}}}+ \\
& +r V_{a}(t)(1-S F) e^{-\frac{\log (2)}{T_{1 / 2}}}
\end{aligned}
$$

where $T_{1 / 2}$ and $t_{r}$ are the dead-cell reabsorption half-time [18] and the time of the last irradiation prior to present day $t$, respectively. The last model dynamic equation represents the oxygenation update by a Gompertz law. Being $\widehat{p O}_{2}$ the maximum achievable level of oxygenation, we defined a time variant asymptotic value $\omega=\widehat{p O}_{2}\left(\frac{k-V_{a}(t+1)}{V_{a}(0)}\right)$. Introducing a constant $\mathrm{pO}_{2}$ variation rate $\lambda$, the dynamics can be written as:.

$p O_{2}(t+1)=\omega e^{\log \left(\frac{p O_{2}(t)}{\omega}\right) e^{-\lambda}}$

The radiosensitivity parameter $\alpha$ is eventually related to the oxygenation level by a linear function as:

$\alpha(t)=\alpha_{\min }+\frac{\left(\alpha_{\max }-\alpha_{\min }\right)}{\widehat{0}_{2}} p O_{2}(t)$

where $\alpha$ ranges between $\alpha_{\min }=0.01 \mathrm{~Gy}^{-1}$ and $\alpha_{\max }=0.5 \mathrm{~Gy}^{-1}$, set according to the literature [18][21]. In conclusion, the complete model encompasses a set of six free parameters (PS), namely $\rho, k, r, \lambda, p O_{2}(0), T_{1 / 2}$.

\section{EXPERIMENTS}

\section{A. Protocol}

A parameter set for each patient group (G1, G2, G3) and the complete data cohort was defined as PS1, PS2, PS3 and PSa, respectively (cfr. section III.A). Among the 16 patients, one patient for each group was kept out from training as outlined in Table I and used for testing and adaptation (cfr. section II.B). PS1 was trained on five patients, namely P1, P2, P3, P4, P6, while P5 was left out due to its larger dataset. PS2 and PS3 were trained on four patients each (P7, P8, P10, P11 and P12, $\mathrm{P} 13, \mathrm{P} 14, \mathrm{P} 15$, respectively). $\mathrm{P} 9$ was randomly chosen as testing patient for G2, whereas P16 was selected among G3 patients according to its larger amount of contoured volumes.

\section{B. Group specific optimization (training data)}

The estimation of each PS was iteratively optimized by means of a custom genetic algorithm implemented in Matlab (Mathworks, USA). At time instant $t=0$, the algorithm started with a population of 300 randomly generated PS. The equation system was solved assuming one day as the time step. Let $n$ and $m$ be the index of the patient and the number of considered measured tumor volumes for that patient, respectively. The difference between the $m$-th measured volume $\bar{v}_{n, m}$ and the corresponding predicted volume $v_{n, m}$ is the fitting error $e_{n, m}$. Considering all the measured volumes $\left(M_{n}\right)$ for the $n$-th patient, the prediction error, can be expressed as:

$$
e_{n}=\sqrt{\frac{\sum_{m}^{M_{n}} e_{n, m}^{2}}{M_{n}}}
$$

The average $e_{n}$ obtained on all the patients included was the fitness corresponding to the tested $P S$ in the optimization. According to the fitness, a $P S$ subset was selected to undergo cross-over (rate: 0.4) and mutation (rate: 0.35), and to 
generate the $P S$ population for the next iteration step. In order to have a more homogenous indicator of the model performance despite the differences in volume sizes across patients, we also computed the percentage fitting error with respect to the volume acquired at the first irradiation day $\left(e_{n} \%=100 e_{n} / v_{n, 1}\right)$. The parameter learning was iterated until the variability of the estimated parameter values, computed across a short iteration span (25), was lower than $1 \%$. In order to avoid premature convergence, at least 200 iterations were imposed. In order to avoid inconsistent results, all the free parameters were bounded appropriately. The carrying capacity value $(k)$ was limited within $100 \%$ and $150 \%$ of the initial tumor volume as non-small tumor mass and proximity to maximal volume extent were assumed.

The growth rate $(\rho)$ was allowed to span in the range $(0.05$, $0.5)$ corresponding to a maximum $10 \%$ increase after 1 day, considering the average initial tumor size of $23 \mathrm{~cm}^{3}$ and $k=1.5 \mathrm{~V}(0)$. The percentage of instantaneously killed cells $(r)$ was bounded in between 0.5 , corresponding to $50 \%$ delayed cell death, and 1 , where only instantaneous cell deaths occur, as in the standard LQ model. The span $0<p O_{2}(0)<100 \%$ was selected to represent all the possible levels in between anoxic and fully oxygenated tumor whereas a $0.05<\lambda<0.5$ range was set to cope with the large variety of mechanisms affecting the reoxygenation process. Finally, the range $\left(1<T_{1 / 2}<10\right)$ of the half-time constant for the dead cell clearance was set according to literature [18] and our preliminary analyses [21].

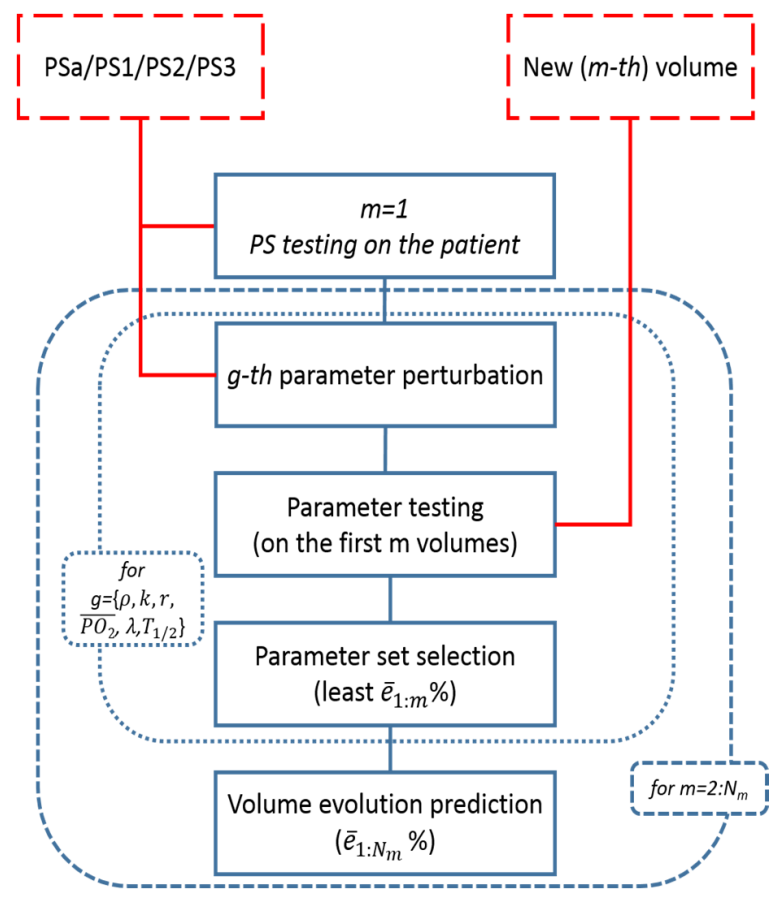

Fig. 2. Model adaptation scheme. The procedure is iterated on the segmented volumes of each patient $(5,9,16)$ and the new parameter set is obtained by perturbing each of the 6 parameters $(\mathrm{g})$ up to $25 \%$ of its range around its optimal value and testing the obtained parameter combination on the first $m$ volumes. An index of the prediction quality is computed by comparing the predicted volumes and the CBCT segmentations.

\section{Model adaptation (testing data)}

The idea underlying the adaptive approach is that the groupspecific optimization allows the definition of a PS close to the best performing one, for all the patients featuring the same tumor type and treatment modalities. Therefore, the patient specific parameter tuning did no longer require a global optimization by means of an evolutionary approach (Fig. 2).

Each PS was used as the initial guess for the adaptive model in order to predict the tumor volume evolution of patients P5, P9 and P16. The on-line adaptation was performed by perturbing every parameter up to $\pm 10 \%$ of its admissible range around the initial guess. Five different values were allowed for each of the 6 parameter $(-10 \%,-5 \%, 0 \%,+5 \%,+10 \%)$ given that the previously defined boundaries were not exceeded. Consequently, about $5^{6}$ combinations were tested iteratively on the first $m$ volumes of the analyzed patient. At each iteration a new volume $(m+1)$ was taken into account. The adaptive algorithm is shown in Fig. 2 . The first step $(m=1)$ corresponds to the initialization of the model to the first acquired volume size (standard model testing). From $m=2$ on, every combination of perturbed parameter (PSj) was tested and the corresponding prediction error computed. The combination $(\widehat{P S})$, leading to the smallest error $\left[e_{n, j}\right]_{1: m}$ across the $m$ considered volumes of patient $n$, was selected. Finally, the prediction error $\left[e_{n, j}\right]_{1: N_{m}}$ of $\widehat{P S}$ on the complete dataset of patient $n$ was computed.

\section{RESULTS}

\section{A. Group-specific optimization}

The parameter training performed on a patient group basis resulted in quite different parameter values across the three subgroups (G1, G2 and G3). All the three parameter sets (PS1, PS2 and PS3) showed similar carrying capacity close to the lower bound (Table II). PS1 and PS2 featured a similar $\rho$ value, greater than about five times the value assessed in PS3. Apparently, G2 was more influenced by the delayed radiation effect $(r=0.5)$, followed by PSa $(r=0.91)$, whilst G1 and G3 showed just instantaneous killing effect $(r=1)$. Since the introduction of $r$ was a novelty with respect to previous attempts to model the delayed response to therapy [21] [22] [23], we analyzed its effect independently of other parameters.

The evolution of a $23 \mathrm{~cm}^{3}$ tumor corresponding to the average initial volume of the 16 patients was allowed to evolve using PSa setting except for the $r$ value, which was set to each of its boundary values ( $r=0.5$ and $r=1)$. The first two weeks of a standard irradiation treatment, administered 5 days per week (dose: 2Gy/fraction), was simulated (Fig. 3). As expected, $r=0.5$ corresponded to a smoother overall volume evolution and a delayed shrinkage. In any case, the gap between the two curves ( $r=1$ and $r=0.5$ ) was rapidly filled during the treatment stop of the week ends. Finally, G2 was the group with the lowest initial oxygenation level $(23 \%)$ with respect to the two others, which had values close to $100 \%$. PSa was the parameter combination resulting in the poorest fitting performance $(e \%>21 \%)$; conversely PS3 showed a promising average error 
$(e \%<14 \%)$ as summarized in Table II.

\section{B. Model adaptation}

When the model adaptation algorithm was initialized by means of the PS optimized on the same group of the testing patient, it resulted in a maximum prediction error less than $10 \%$ for all the three patients $(5,9$, and 16 ) already at $m=1$ (no parameter adjustment occurring). As far as the general model (PSa) is concerned, it resulted in a larger initial error (15-20\%), but still comparable to the fitting errors (cfr. Table II).

Along the adaptation process, the performance curves were not strictly monotone, as shown in Fig. 4. In particular, the error evolution for the PSa initialized adaptation tested on patient 16 (Fig. 4, panel c), shows two ripples causing an error doubling when $m=2$ and $m=6$ volumes are encompassed in the adaptation. Interestingly, after the inclusion of a number of volumes corresponding to about the first two treatment weeks ( $\sim 10$ contours), the gap between the two approaches was significantly shortened, no more ripples were found, and the error was always close or below the $10 \%$ threshold. In other words, the PS adaptation procedure always equals or improves the model prediction abilities for $m \geq 10$. When all the contours were included in the adaptation $\left(m=N_{m}\right)$, the model error with respect to the measured volume was between $5 \%$ and $10 \%$ independently from the initial PS selected. Only in the case of patient 9, the group performance (PS2) did result in a significantly more accurate prediction $(e \%=5.5 \%)$ with respect to the PSa $(e \%=7.2 \%)$. A few examples of different volume prediction curves are shown for patient 5 in Fig. 5. It can be observed that while the straightforward application of PSa causes an overestimation of the tumor volume size, the tuned version of the parameter combination $(m=10)$ is a good representation of patient 5 tumor progression. Conversely, PS1 performance mimicking patient 5 volume evolution is almost independent from the number of volumes included in the adaptation.

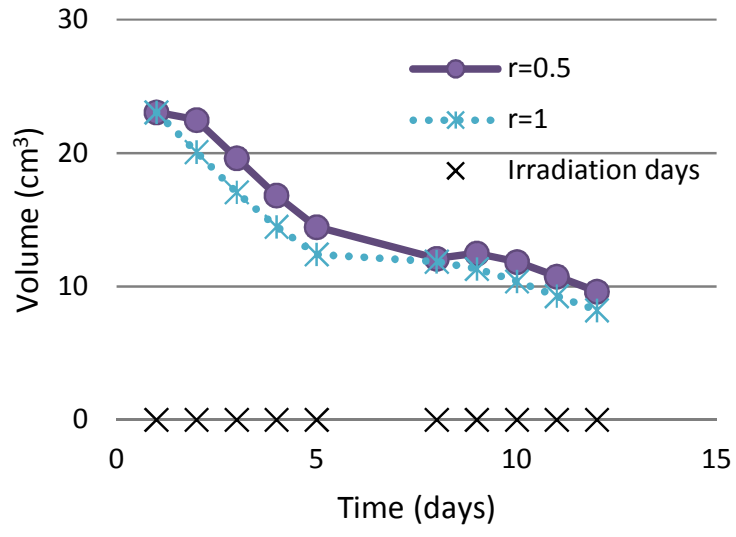

Fig. 3. Simulation of the effects of different $r$ settings. The solid line represents overall volume shrinkage in case of $50 \%$ of cell death delayed, while the dotted line is obtained simulating instantaneous cell killing only. Crosses at the baseline indicate irradiation days in a realistic treatment plan: 5 fraction/week, 2Gy/fraction.
TABLE II.

GROUP SPECIFIC OPTIMIZATION: PARAMETER VALUES AND FITTING PERFORMANCES ON THE TRAINING PATIENT SETS. THE RMS ERROR $\boldsymbol{e}$ IS EXPRESSED IN $\mathrm{CM}^{3}$

\begin{tabular}{ccccccccc}
\hline \hline & $\boldsymbol{\rho}$ & $\boldsymbol{k} \%$ & $\boldsymbol{p} \boldsymbol{O}_{\mathbf{2}}(\mathbf{0})$ & $\boldsymbol{\lambda}$ & $\boldsymbol{r}$ & $\boldsymbol{T}_{\mathbf{1} / \mathbf{2}}$ & $\boldsymbol{e}$ & $\boldsymbol{e} \%$ \\
\cline { 2 - 9 } PS1 & 0.27 & 101.6 & 99 & 0.075 & 1.00 & 1 & 2.8 & 14.3 \\
PS2 & 0.21 & 100.1 & 29 & 0.050 & 0.50 & 1 & 3.9 & 17.7 \\
PS3 & 0.05 & 100.1 & 99 & 0.052 & 1.00 & 10 & 3.9 & 13.6 \\
PSa & 0.25 & 100.1 & 53 & 0.050 & 0.91 & 1 & 4.8 & 21.1 \\
\hline \hline
\end{tabular}
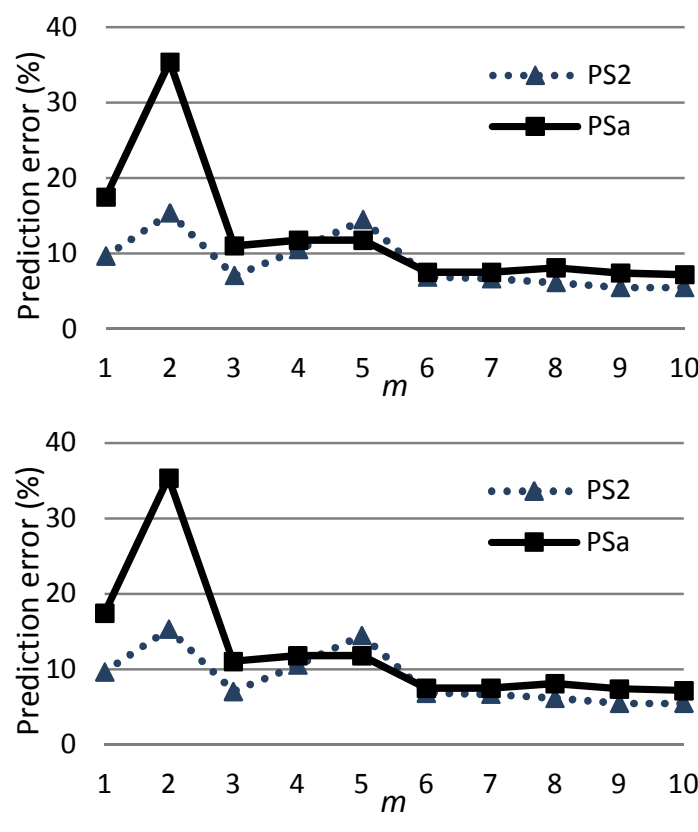

(a)

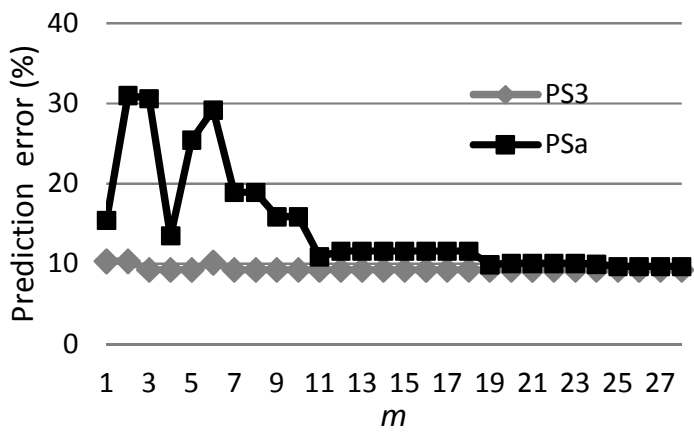

(b)

Fig. 4. Model adaptation comparison. Each panel shows the model adaptation performance $(e \%)$ as a function of the number of volumes $(m)$ considered in the adaptation process for patient 5 (a), 9 (b) and 16 (c), respectively. The continuous line represent the results using PSa while the dashed/dotted PS1, PS2 and PS3, respectively.

\section{DISCUSSION}

\section{A. Major findings}

In this paper, we propose a macroscopic model of tumor growth and radiotherapy response able to predict cancer evolution during treatment. Model parameters were assessed using CBCT volume data in 16 patients, affected by uterine cervical cancer. In a previous work [21], we showed that 
patient-specific models cannot be easily generalized to other patients. In our present work, we investigated whether both general and group-specific models have sufficient extrapolation ability, and proposed a novel adaptation schema that allows the refinement of the model parameters on an individual basis on-the-fly as soon as the volumetric tumor data are available along the treatment course.

Preliminary results about the limited predictive ability of the general model PSa suggest that patient inclusion criteria play a key role. This was confirmed by the results of group-specific models (fitting errors: 13-18\%, intra-group prediction accuracy: 90\%), allowing one to reasonably argue that an appropriate stratification of the training patients is a key factor to achieve a reasonable agreement between prediction and experimental data.

The parameter adaptation of the PSa model reduced the prediction error of about one-half with respect to its initial value (error range: $20-15 \%$ ) reaching promising performance (error range: $7-10 \%$ ). These results, comparable to the fitting performance (mean error: $\sim 5 \%$ ) of the patient-specific parameter optimization investigated in our previous study [21], suggest that this novel approach can successfully customize the group-specific model. Moreover, a similar error range (7-12\%) was found when the adaptation is stopped after about 10 iterations, and its trend is always monotone and decreasing afterward. This suggests that the advantage of the model tuning is the increased accuracy and reliability provided after about two weeks from the first radiotherapy session, as opposed to the unreliable generalization ability of the patientspecific approach [21].

Interestingly, the novel adaptive approach represents a safer alternative in case of uncertainties in the parameter setting, as shown by the PSa results, and it was developed in order to be easily applicable to a real clinical scenario. It was inspired by the IGRT paradigm, where adjustments in dose delivery are triggered by tumor motion and morphological changes that can be observed on clinical images [4] [7]. Our adaptive model, however, aims at predicting patient-specific variation in response to radiation at a macroscopic level, which could early provide information on the treatment outcome and, consequently, warning about the need for therapy adjustment (e.g., schedule, dose per fraction).

A 3D model featuring fine spatial description, such as the one presented in [13], could be created according to the macroscopic prediction of our simpler system trained on actual clinical data, in order to provide a comparable overall tumor evolution. This approach has already been suggested in the literature comparing, for example, the performance of an isomorphic and a $3 \mathrm{D}$ statistical model adjusted to reproduce similar volume sizes [25]. The adaptive approach integrated with a morphological description of the tumor evolution could theoretically allow for increased accuracy of the treatment delivery and reduction of the healthy tissue toxicity. A reliable description at a more complex and detailed level would require the introduction of further information (e.g., PET, MRI and histology).

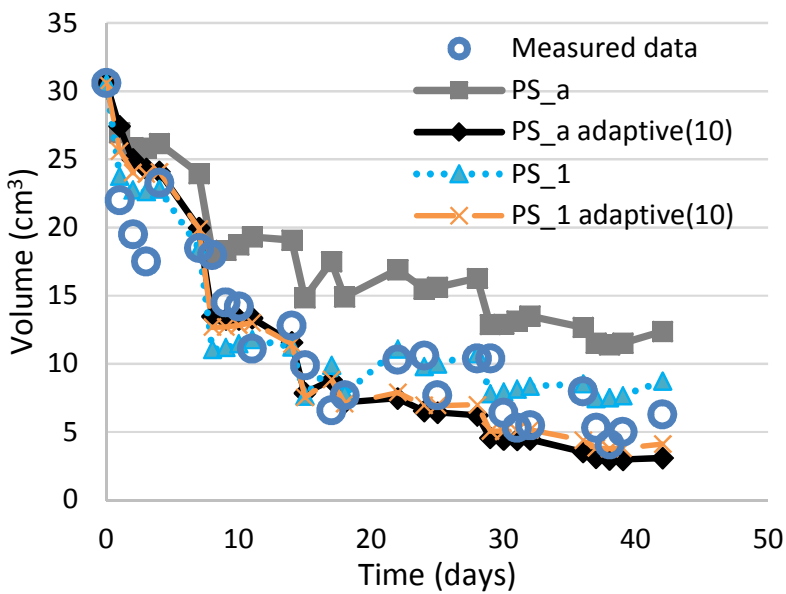

Fig. 5. Model adaptation comparison for patient 5. Volume evolution curves, predicted using PSa and PS1 before the parameter perturbation $(m=1)$ and after 10 steps of the adaptation process are displayed along with the GTV values computed from the segmented CBCT (circles)

\section{B. Modeling issues}

Despite the promising results, some issues have to be outlined and require further discussion, namely: 1) data uncertainty; 2) limited patient cohort; 3) genetic algorithm convergence; 4) nominal dose; 5) lack of independent validation for the oxygenation and necrotic volume dynamics.

We acknowledge that CBCT images feature suboptimal contrast enhancement for soft tissues; nevertheless, they are widely used in common clinical practice [28]. The objective evaluation of the segmentation inter-observer variability was beyond the scope of the present work. A preliminary test on patient P1 data, involved a threefold tumor segmentation, performed by the same expert oncologist at a time distance of approximately one month from one each other. The result was a $10 \%$ variability in the volume calculations. We plan to further investigate this issue by combining data deriving from different imaging techniques (e.g. MRI), and by involving more expert observers.

Only 16 patients were included in the study and the subdivision into three groups, leaving three patients out for optimization purposes, caused PS1, PS2 and PS3 optimization to be based on 5, 4 and 4 patients, respectively. The limited data cohort is likely to have affected the parameter learning and prevented a more specific classification taking into account tumor staging along with type and treatment. Nevertheless, it has to be considered that, over 150 CBCT volumes were analyzed, and the results showed a nice prediction ability of the model, with the group specific approach outperforming the general formulation PSa.

Another issue that might affect a reliable parameter estimation is the convergence stability of the custom genetic algorithm. We introduced a minimum number of iterations (200) in order to limit such inconvenient (cfr. par. III.B). Moreover, we repeated the optimization 10 times on P5, P9 and P16, separately, and observed that different random initialization may lead to different parameter setting. 
However, the maximum error variation across the 10 optimizations was always lower than $1 \%$ (max error $<7.5 \%$ ). In one patient (P5) the maximum error spread was limited within $0.02 \%$. In light of the $10 \%$ uncertainty found in the CBCT data, all the combinations found may be considered as equally valid. Therefore, the volume segmentation variability remains the most limiting factor. Nevertheless, a possible refinement or replacement of the genetic algorithm should be addressed in further studies.

The dose administered to the tumor may not correspond to the prescribed one due to tumor displacement and shrinkage along the treatment course [28]. A co-registration of CBCT images and the computation of the actual delivered dose is expected to improve the model accuracy. At present our assumption about the agreement between nominal and actual dose, relies on the fact that two extra margins were included in the treatment plan. The clinical target volume (CTV) aims at accounting for the invisible tumor micro-infiltration, while the planning target volume (PTV) allows for uncertainties in planning or treatment delivery (cfr. isodose profile, Fig. 1).

A quite complex issue is related to the lack of independent validations for all the four proposed dynamics (active, inactive, and necrotic volumes, along with oxygen). There can be several parameter combinations able to explain the same overall tumor size evolution. For example, a slow shrinkage could be due both to a low radio-sensitivity (possibly related to hypoxia) or to a large dead cell clearance time. Therefore, the relative percentage of the necrotic volume and the role of oxygenation should be investigated further. For example, the inclusion of functional information such as blood-oxygenlevel dependent (BOLD) MRI or MRI-based oxygen imaging (MOXI) could provide quantitative or semi-quantitative information about tumor oxygenation, in an entirely noninvasive way [29] [30]. Moreover, this would allow us to verify the hypothesized relationships among $p O_{2}$ level, tumor radio-sensitivity, and volume size.

\section{CONCLUSION}

In conclusion, this work was able to achieve the following outcomes: a) a novel macroscopic model able to include active, inactive and necrotic tumor volume evolution along with the oxygenation dynamics was proposed; b) the group specific training resulted in a fitting error within $13 \%$ and $18 \%$ for the three subgroups separately and $21 \%$ for the complete data cohort; c) their extrapolation abilities were tested and it was found that PS1, PS2 and PS3 reached better prediction performance on patient 5, 9 and 16 (about 9\%, 10\% and 10\%, respectively) than PSa (about 20\%, 17\% and 15\%, respectively); d) the adaptation procedure was able to successfully improve PSa prediction, making it comparable to the ones of the other subgroups, within about 10 iterations.

We believe that, despite the limited data cohort, the promising results achieved by means of the adaptive approach represent a new step toward the translation of mathematical predictive models into clinical practice. The long term goal is the exploitation of in-silico prediction to support the decision making process in the treatment planning phase. Similarly, validated prediction models would help the physician to adapt the therapy administration along the course of treatment to patient specific response, using the available imaging data.

\section{REFERENCES}

[1] B.W. Stewart and C.P. Wild. (eds). "World Cancer Report 2014". International Agency for Research on Cancer. World Health Organization, 2014.

[2] N. Howlader, A.M. Noone, M. Krapcho, J. Garshell, D. Miller, S.F. Altekruse, C.L. Kosary, M. Yu, J. Ruhl, Z. Tatalovich, A. Mariotto, D.R. Lewis, H.S. Chen, E.J. Feuer and K.A. Cronin (eds). "SEER Cancer Statistics Review". 1975-2011, National Cancer Institute. Bethesda, MD, 2014.

[3] N. Colombo, S. Carinelli, A. Colombo, C. Marini, D. Rollo and C. Sessa, "Cervical cancer: ESMO clinical practice guidelines for diagnosis, treatment and followup". Annals of Oncology, vol. 23, suppl. 7, pp. vi27-vi32, 2012.

[4] R. Jadon, C.A. Pembroke, C.L. Hanna, N. Palaniappan, M. Evans, A.E. Cleves and J. Staffurth, "A Systematic Review of Organ Motion and Image-guided Strategies in External Beam Radiotherapy for Cervical Cancer". Clin Oncol (R Coll Radiol), vol. 26, no. 4, pp. 185-96, 2014.

[5] M.L. Bondar, M. Hoogeman, J.W. Mens, G. Dhawtal, I. de Pree, R. Ahmad, S. Quint and B. Heijmen, "Toward an individualized target motion management for IMRT of cervical cancer based on model-predicted cervixuterus shape and position". Radiotherapy \& Oncology, vol. 99, no. 2, pp. 240-245, 2011.

[6] M.L. Bondar, M.S. Hoogeman, J.W. Mens, S. Quint, R. Ahmad, G. Dhawtal and B.J. Heijmen, "Individualized nonadaptive and online-adaptive intensity-modulated radiotherapy treatment strategies for cervical cancer patients based on pretreatment acquired variable bladder filling computed tomography scans". International Journal Radiation Oncology Biology Physics, vol. 83, no. 5, pp. 1617-1623, 2012.

[7] A. Nagano, S. Minohara, S. Kato, H. Kiyohara and K. Ando, "Adaptive radiotherapy based on the daily regression of a tumor in carbon-ion beam irradiation" Phis. Med. Biol., vol. 57, no. 24, pp. 8343-56, 2012.

[8] R.W. Tsang, A.W. Fyles, P. Kinrkbride, W. Levin, L.A. Manchul, M.F. Milosevic, G.A. Rawlings, D. Banerjee, M. Pintilie and G.D. Wilson, "Proliferation measurements with flow cytometry Tpot in cancer of the uterine cervix: correlation between two laboratories and preliminary clinical results". Int. J. Radiat. Oncol. Biol. Phys., vol. 32, pp. 1319-1329, 1995.

[9] J.P. Banàth, S.H. MacPhail and P.L. Olive, "Radiation sensitivity, H2AX phosphoilation, and kinetics of repair of DNA strand breaks in irradiated cervical cancer cell lines". Cancer Research, vol. 64, no.19 pp. 7144-7149, 2004.

[10] J. T. Oden, A. Hawkins and S. Prudhomme, "General diffuse-interface theories and an approach to predictive tumor growth modeling." Mathematical Models and 
Methods in Applied Sciences, vol. 20, no. 3, pp. 477-517, 2010.

[11] H-H. Dubben, H.D. Thames and H-P. Beck-Bornholdt, "Tumor volume: a basic and specific response predictor in radiotheraphy". Radiotherapy and Oncology, vol. 47, no. 2, pp. 167-174, 1998.

[12] R.R. Hallac, Y. Ding, Q. Yuan, R.W. McColl, J. Lea, R.D. Sims, P.T. Weatherall and R.P. Mason, "Oxygenation in cervical cancer and normal uterine cervix assessed using blood oxygenation level-dependent (BOLD) MRI at 3T". NMR Biomed., vol. 25 pp. 1321-1330, 2012.

[13] B. Titz and R. Jeraj, "An imaging-based tumour growth and treatment response model: investigating the effect of tumour oxygenation on radiation therapy response," Phys. Med. Biol., vol. 53, pp. 4471-4488, 2008.

[14] C. Harting, P. Peschke and C.P. Karger, "Computer simulation of tumour control probabilities after irradiation for varying intrinsic radio-sensitivity using a single cell based model". Acta Oncol., vol. 49, no. 8, pp. 1354-1362, 2010.

[15] G. Stamatakos, D. Dionysiou, A. Lunzer, R. Belleman, E. Kolokotroni, E. Georgiadi, M. Erdt, J. Pukacki, S. Rueping, S. Giatili, ' A. d' Onofrio, S. Sfakianakis, K. Marias, C. Desmedt and N. Graf, "The technologically integrated oncosimulator: combining multiscale cancer modeling with information technology in the in silico oncology context." Biomedical and Health Informatics, IEEE Journal of, vol. 18, no. 3, pp. 840-854, 2014.

[16] K. Lim, P. Chan, R. Dinniwell, A. Fyles, M. Heider, Y. Cho, D. Jeffray, L. Manchul, W. Levin, R.P. Hill and M. Milosevic, "Cervical cancer regression measured using weekly magnetic resonance imaging during fractionated radiotherapy: Radiobiologic modeling and correlation with tumor hypoxia”. Int. J. Radiat. Oncol. Biol. Phys., vol. 70, no. 1, pp. 126-133, 2008.

[17] R. Rockne, J. K. Rockhill, M. Mrugala, A.M. Spence, I. Kalet, K. Hendrickson, A. Lai, T. Cloughesy, E.C. Alvord Jr and K.R. Swanson, "Predicting the efficacy of radiotherapy in individual glioblastoma patients in vivo: a mathematical modeling approach". Phys. Med. Biol., vol. 55, pp. 3271-3285, 2010.

[18]Z. Huang, N.A. Mayr, W.T. Yuh, S.S. Lo, J.F. Montebello, J.C. Grecula, L. Lu, K. Li, H. Zhang, N. Gupta and J.Z. Wang, "Predicting outcomes in cervical cancer: a kinetic model of tumor regression during radiation therapy". Cancer Research, vol. 70, no. 2, pp. 463-70, 2010.

[19] M.J. Piotrowska, U. Foryś, M. Bodnar, J.A. Poleszczuk "Simple model of carcinogenic mutations with time delay and diffusion". Mathematical Biosciences and Engineering, vol. 10, no. 3, pp.861-872, 2013.

[20] P. Wang and Y. Feng, "A mathematical model of tumor volume changes during Radiotherapy". Scientific Work Journal , 2013

[21] A. Belfatto, M. Garbey, M. Riboldi, D. Ciardo, A. Cecconi, R. Lazzari, B.A. Jereczek, R. Orecchia, G. Baroni and P. Cerveri, "Modeling Cervix Cancer Growth and Response to Radiation Therapy: A Validation Study
Using Patient Volumetric Tumor Data”. Biomedical and Health Informatics (BHI), 2014 IEEE-EMBS International Conference on. IEEE, pp. 476-479, 2014.

[22] A. Belfatto, M. Riboldi, D. Ciardo, F. Cattani, A. Cecconi, R. Lazzari, B.A. Jereczek, R. Orecchia, G. Baroni and P. Cerveri, "Kinetic Models for Predicting Cervical Cancer Response to Radiation Therapy on Individual Basis Using Tumor Regression Measured In Vivo With Volumetric Imaging." Technology in cancer research \& treatment, 2015. DOI: 1533034615573796.

[23] A. Belfatto, M. Riboldi, G. Baroni, D. Ciardo, F. Cattani, A. Cecconi, R. Lazzari, B.A. Jereczek, R. Orecchia, and P. Cerveri. "Modeling the interplay between tumor volume regression and oxygenation in uterine cervical cancer during radiotherapy treatment." Journal of Biomedical and Health Informatics, 2015. DOI: 10.1109/JBHI.2015.2398512

[24]E.C. Georgiadi, D.D. Dionysiou, N. Graf and G.S. Stamatakos, "Toward in silico oncology: Adapting a four dimensional nephroblastoma treatment model to a clinical trial case based on multi method sensitivity analysis". Computers in Biology and Medicine, vol. 42, no. 11, pp. 1064-78, 2012.

[25] Y. Liu, T.C. Chan, C.G. Lee, Y.B. Cho and M.K. Islam, "A stochastic model for tumor geometry evolution during radiation therapy in cervical cancer". Med. Phys., vol. 41, no. 2, 2014. DOI: $10.1118 / 1.4859355$.

[26] M. Hadjiandreou and G. Mitsis, "Mathematical modeling of tumor growth, drug-resistance, toxicity, and optimal therapy design". IEEE Trans Biomed Eng., vol. 61, no. 2, pp. 415-425, 2014.

[27] K. Kum Kum and S.P. Jackson. "DNA double-strand breaks: signaling, repair and the cancer connection". Nature genetics, vol. 27, no. 3, pp. 247-254, 2001.

[28] N. Tyagi, J.H. Lewis, C.M. Yashar, D. Vo, S. Jiang, B., A.J. Mundt and L.K. Mell, "Daily online cone beam computed tomography to assess interfractional motion in patients with intact cervical cancer". International Journal of Radiation Oncology Biology Physics, vol. 80 no. 1, pp. 273-280, 2008.

[29] R.R. Hallac, Y. Ding, Q. Yuan, R. W. McColl, J. Lea, R.D. Sims, P.T. Weatherall, and R.P. Mason, "Oxygenation in cervical cancer and normal uterine cervix assessed using blood oxygenation level-dependent (BOLD) MRI at 3T”. NMR Biomed., vol. 25, no. 12, pp. 1321-1330, 2012.

[30]Z. Zhang, R.R. Hallac, P. Peschke, and R.P. Mason, “A noninvasive tumor oxygenation imaging strategy using magnetic resonance imaging of endogenous blood and tissue water". Magnetic Resonance in Medicine, vol. 71, no. 2, pp. 561-569, 2014. 